\title{
Working memory maintenance contributes to long-term memory formation: Evidence from slow event-related brain potentials
}

\author{
Patrick Khader \\ Philipps University Marburg, Marburg, Germany \\ Charan Ranganath \\ University of California, Davis, California \\ AND \\ Anna Seemüller and Frank Rösler \\ Philipps University Marburg, Marburg, Germany
}

\begin{abstract}
Behavioral research has led to conflicting views regarding the relationship between working memory (WM) maintenance and long-term memory (LTM) formation. We used slow event-related brain potentials to investigate the degree to which neural activity during WM maintenance is associated with successful LTM formation. Participants performed a WM task with objects and letter strings, followed by a surprise LTM test. Slow potentials were found to be more negative over the parietal and occipital cortex for objects and over the left frontal cortex for letter strings during WM maintenance. Within each category, they were enhanced for items that were subsequently successfully remembered. These effects were topographically distinct, with maximum effects at those electrodes that showed the maximum negativity during WM maintenance in general. Together, these results are strongly consistent with the ideas that WM maintenance contributes to LTM formation and that this may occur through strengthening of stimulus-specific cortical memory traces.
\end{abstract}

A great deal of research in cognitive neuroscience has investigated the neural mechanisms of processes that support the active maintenance of information in working memory (WM) and the encoding and retrieval processes that support long-term memory (LTM). However, these topics are typically examined in isolation, and little is known about whether or how these processes might interact. For example, it is currently unclear how WM maintenance (i.e., the ability to keep a stimulus in mind for a limited time period) affects new LTM formation.

Historically, this has been a topic of considerable debate in cognitive psychology. For example, Hebb (1949) suggested that the transient maintenance of information by means of reverberating activity in specialized neural cell assemblies serves to strengthen LTM traces in the brain. In the 1960s, many models (e.g., Atkinson \& Shiffrin, 1968) proposed that the amount of time spent actively maintaining an item directly determines the degree to which it will be remembered later. However, some studies of this prediction yielded inconclusive results concerning the relationship between rote WM maintenance and LTM (Craik \& Watkins, 1973; Woodward, Bjork, \& Jongeward, 1973) and found that successful recall depends more on the depth of processing of an experienced stimulus (Craik,
2002; Craik \& Lockhart, 1972). On the basis of these and other findings, some researchers have suggested that rote WM maintenance and LTM formation might be functionally independent (Craik \& Lockhart, 1972).

Until recently, psychological and neurophysiological studies on this topic have assumed that maintenance is supported by a unitary WM process. However, some findings (Johnson, Reeder, Raye, \& Mitchell, 2002; Jolicœur \& Dell'Acqua, 1998; Naveh-Benjamin \& Jonides, 1984a, 1984b; Raye, Johnson, Mitchell, Reeder, \& Greene, 2002) suggest that WM maintenance may consist of at least two separate stages. In the first stage, recently encoded sensory stimuli are transformed into representational codes (e.g., articulatory, phonological, visual, oculomotor) that can be maintained in the absence of external stimulation. ${ }^{1}$ Once these codes are established, a second, more automated stage of rehearsal can proceed. The early stage of WM maintenance may be thought of as relatively phasic (see, e.g., Johnson et al., 2002), whereas the late stage likely persists throughout the remaining time that an item is maintained. This notion follows from findings showing that the early and late portions of a maintenance rehearsal sequence could be discriminated according to their degree of automaticity. For example, Naveh-Benjamin and

P. Khader, khader@staff.uni-marburg.de 
Jonides (1984b) showed that participants found it more difficult to interrupt overt utterances of a rehearsal sequence of words during the later part of a rehearsal period. Furthermore, they showed that the initial stage of rehearsal demands more processing resources than the later stage, because participants took longer to detect a probe stimulus during the later rehearsal interval.

Critically, recent evidence strongly suggests that the early stage of WM maintenance disproportionately promotes successful LTM formation relative to the later stage (Johnson et al., 2002; Naveh-Benjamin \& Jonides, 1984a, 1984b). For example, Naveh-Benjamin and Jonides (1984a) showed that the first few rehearsals in a sequence of words had an effect on later recognition performance, whereas later rehearsals had a relatively small effect (see Naveh-Benjamin \& Jonides, 1984b, for related evidence). Furthermore, Ranganath, Cohen, and Brozinsky (2005) demonstrated that interfering with processing during the initial stage of WM maintenance impaired LTM formation. In this study, participants actively maintained a novel, 3-D visual object, and on some trials an array of lines was shown during the memory delay. When the array was shown, participants were instructed to verbally indicate the number of lines shown in the array. Performing the secondary task lowered the number of subsequently remembered objects only if the line arrays were presented during the initial part of the delay period. Presentation of distractor arrays later in the delay had no effect on subsequent LTM performance. These findings suggest that processing during the initial stage of WM maintenance can be functionally distinguished from subsequent maintenance processing. Furthermore, processing during the initial stage of WM maintenance may disproportionately promote subsequent LTM formation.

If WM maintenance indeed supports LTM formation, neural activity during WM maintenance should be predictive of subsequent LTM performance. Consistent with this hypothesis, results from an fMRI study showed that activity in the dorsolateral prefrontal cortex, occipital cortex, and hippocampus during the initial stage of WM maintenance was predictive of subsequent LTM performance (Ranganath et al., 2005). These and related findings (Blumenfeld \& Ranganath, 2006; Davachi, Maril, \& Wagner, 2001; Schon, Hasselmo, LoPresti, Tricarico, \& Stern, 2004) strongly support the idea that neural activity, specifically during the initial stage of WM maintenance, promotes LTM formation. However, the sluggishness of the hemodynamic response measured by fMRI raises questions regarding whether activity related to sensory, perceptual, and attentional processing of an external stimulus can truly be dissociated from responses related to processes occurring during the early delay period (i.e., because the two hemodynamic responses should overlap to a substantial degree; Zarahn, Aguirre, \& D'Esposito, 1997).

In the present study, we sought to further substantiate the claim that WM activity promotes LTM encoding by using electroencephalography (EEG) to monitor the relationship between neural activity, WM maintenance, and subsequent LTM recall. Specifically, we recorded early event-related brain potentials (ERPs) as well as late event- related shifts in the EEG (ranging from $500 \mathrm{msec}$ to several seconds), which are called slow event-related brain potentials (often shortened to slow waves or direct current (DC) potentials; Birbaumer, Elbert, Canavan, \& Rockstroh, 1990). Slow-wave amplitude and topography can be systematically related to cognitive processes such as WM maintenance and LTM retrieval (Rösler, Heil, \& Röder, 1997). Furthermore, slow waves are assumed to arise from synchronized excitatory postsynaptic potentials (EPSPs) at neocortical pyramidal cells arranged perpendicular to the cortical surface (McCallum \& Curry, 1993; Mitzdorf, 1985, 1991; Speckmann \& Elger, 1999). Given that these cortical field potentials are strongly correlated with the hemodynamic response as measured with fMRI (Arthurs \& Boniface, 2002; Logothetis, Pauls, Augath, Trinath, \& Oeltermann, 2001; Mukamel et al., 2005), ERPs and fMRI can provide complementary sources of evidence regarding the relationship between WM and LTM formation. Although ERP recordings have limited spatial resolution in comparison with fMRI, ERPs more directly measure neural activity and have a high temporal resolution in the range of milliseconds. Accordingly, this technique is ideally suited to test the hypothesis that cortical activity occurring specifically during WM maintenance contributes to LTM performance.

A second goal of the present study was to determine the generality of neurocognitive mechanisms that might link WM maintenance to LTM formation. One possibility is that the early stage of WM maintenance involves domain-general control processes that consolidate WM representations (Jolicœur \& Dell'Acqua, 1998), and that the engagement of these control processes contributes to successful LTM formation (Naveh-Benjamin \& Jonides, 1984a, 1984b). An alternative possibility is that WM processes modulate LTM formation through direct effects on specific stimulus representations. Indeed, there is abundant evidence that different cortical regions are activated during maintenance of different kinds of materials (Fuster, 1995; Pasternak \& Greenlee, 2005; Postle, 2006; Ranganath, 2006; Ranganath \& D'Esposito, 2005). At present, it is unclear whether activity in these regions is also linked to LTM.

Here, we used recordings of EEG slow waves to identify the relationship between WM and LTM encoding processes and compared neural activity during processing of verbal and nonverbal stimuli. More specifically, EEG was recorded while our participants performed WM tasks with line drawings of novel 3-D objects and letter strings as stimuli (Figure 1A). Subsequently, they completed a surprise LTM test consisting of items that were seen during the WM task and unstudied foil items. We then examined EEG slow-wave amplitudes during maintenance of objects and letter strings as a function of LTM performance.

With this design, we were able to address two questions. First, we investigated whether neural activity during WM maintenance is predictive of subsequent LTM performance, especially during the early phase of the maintenance delay. If so, we would expect to see maximal ERP differences between subsequently remembered and forgotten items during the memory delay period, especially 


\section{A Examples of Experimental Stimuli}

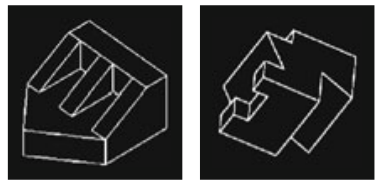

Objects

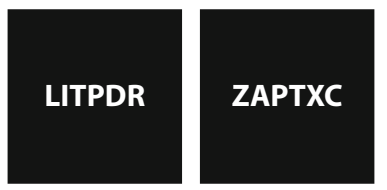

Letter Strings

B Trial Timing

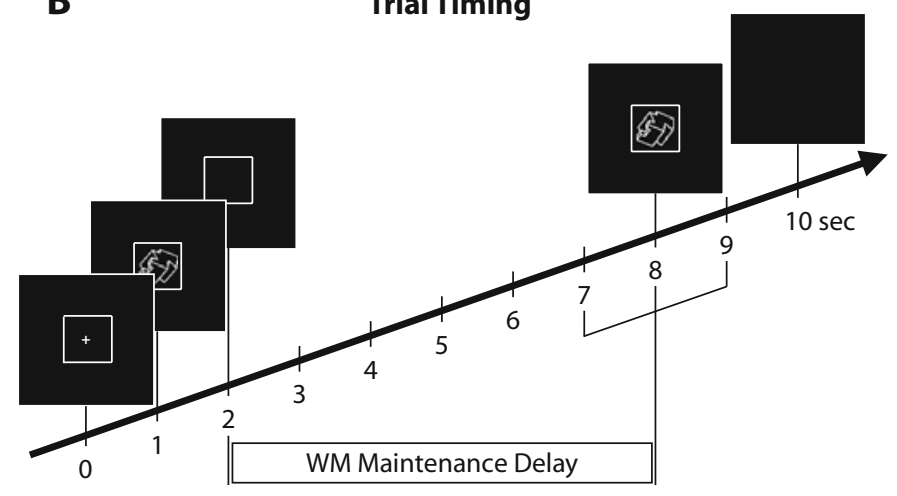

Figure 1. Examples of objects and letter strings that had to be maintained throughout the delay period (A) and trial timing (B). The length of the delay was evenly distributed across trials and ranged from 5 to $7 \mathrm{sec}$, with an average of $6 \mathrm{sec}$.

during the initial part. Second, we tested whether these effects could be observed for both objects and letter strings, and whether their scalp topographies could be differentiated. These analyses allowed us to determine whether WM processing promotes LTM formation through the engagement of generalized control processes or through direct effects on specific stimulus representations that are being maintained. In the former case, one would expect to see qualitatively similar subsequent memory effects for both objects and letter strings, whereas in the latter case one would expect the two effects to be associated with qualitatively distinct scalp topographies.

\section{METHOD}

\section{Participants}

Twenty-seven students at the University of Marburg were recruited. Data of 2 participants had to be excluded due to a program error during the WM phase. Furthermore, for the EEG analysis, those participants for whom fewer than 15 artifact-free EEG segments remained for ERP extraction in either the "remembered" or the "forgotten" condition had to be excluded, yielding 16 participants ( 9 female, mean age $=21.88$ years, $S D=1.82$ ) for the objects and 14 participants ( 7 female, mean age $=22.07$ years, $S D=1.86$ ) for the letter string condition. For comparability with the ERP subsequent memory effects, the behavioral data were also analyzed with these different groups of subjects. Furthermore, only those 12 participants who had been included in both the objects and the letter strings analyses ( 6 female, mean age $=21.83$ years, $S D=$ 1.80 ) were entered into the overall analysis of WM maintenance (independent of whether the stimuli were subsequently remembered or forgotten). All the participants were right-handed, healthy, and native speakers of German. They were naive with respect to the objective of the experiment, gave written consent to participate, and received either monetary compensation or course credit.

\section{Materials}

The object stimuli consisted of 200 line drawings of 3-D novel "possible" objects (see Figure 1A) drawn in part from previous studies (Schacter \& Cooper, 1993; Schacter, Cooper, \& Delaney, 1990; Williams \& Tarr, 1997, 1999). These stimuli have also been used in a previous fMRI study (Ranganath et al., 2005), in which the relationship between visual WM maintenance and LTM formation was examined. To control for variability in size, and to ensure that all objects fit well into the fixation frame (see below), the object pictures were standardized in such a way that the longest side (height or width) was always $4 \mathrm{~cm}$, resulting in widths and heights between 3.5 and $4 \mathrm{~cm}$. The letter strings were 200 capitalized nonwords with a constant length of six letters, consisting of a randomly chosen threeletter syllable (consonant-vowel-consonant) followed by three randomly chosen consonants (see Figure 1A). All letter strings were $0.8 \mathrm{~cm}$ high and $3.5-4 \mathrm{~cm}$ wide.

\section{Procedure}

The participants sat in an electrically shielded and dimly lit experimental chamber facing a computer screen $70 \mathrm{~cm}$ in front of them and performed 200 trials of a delayed matching-to-sample task (see Figure 1B). Trials started with the presentation of a small white fixation cross for $1 \mathrm{sec}$. Then, a cue object or letter string was shown for $1 \mathrm{sec}$, and the participants were instructed to internally rehearse the stimulus across a variable 5- to 7-sec delay period. Following the delay, a probe stimulus (either matching or nonmatching) was shown for $2 \mathrm{sec}$, and the participants pressed one of two keys on a computer keyboard to indicate whether the probe was identical to or different from the cue stimulus. A variable intertrial interval (ITI) of 2-3 sec preceded the start of the next trial. All stimuli were presented in white at the center of a black screen. The participants were instructed to fixate the center of the screen throughout the trial. A gray square $(5 \times 5 \mathrm{~cm})$, visible throughout the trial but not during the ITI, framed the presented stimuli and served as a fixation aid. The participants were instructed to suppress blinks or eye movements while the frame was visible. An equal number of match and nonmatch trials were presented in a random sequence. In the WM 
task, 150 of the 200 objects and 150 of the 200 letter strings were used, with 50 stimuli of each group for the "match" condition, 50 for the "nonmatch" condition, and 50 serving as nonmatching targets in the latter condition. The remaining 50 objects and 50 letter strings were used as new foils in the subsequent memory test. The WM task started with 10 practice trials ( 5 with objects and 5 with letter strings) containing filler stimuli that were not included in the subsequent memory test. Then, the 200 experimental trials were presented in eight blocks (alternating between object and letter string blocks) of 25 items each. After each block, a pause of at least $10 \mathrm{sec}$ was enforced, allowing the experimenter to perform a DC reset of the EEG.

After the EEG recording session, the participants completed a surprise recognition (LTM) test on the items that were presented as cue stimuli during the preceding WM task. The test included all 100 objects and letter strings from the WM task, interspersed with 50 new foil items that had not previously been presented. All stimuli were shown individually on a computer screen, and the participants were instructed to rate each item on a confidence scale that ranged from 1 to 4 (with 1 being definitely seen; 2, probably seen; 3, probably not seen; and 4 , definitely not seen). The stimuli remained on the screen until the participants responded by pressing one of four keys on a computer keyboard. The first 6 participants also had the possibility to select a fifth category termed don't know. However, postexperimental debriefings showed that these participants used this category either rarely or as a weaker version of the probably not seen category. Therefore, we decided to drop this category for the remaining participants and to merge these responses with those in the other not seen categories (see below for a more detailed description of how categories were selected and combined for data analysis).

Between the WM task and the memory test, the Oldfield Handedness Inventory (Oldfield, 1971) was inserted as a distractor task (approximately $10 \mathrm{~min}$ ) to reduce recency effects on memory retrieval.

\section{EEG Recording, Artifact Handling, Signal Extraction, and Statistical Analysis}

The EEG was recorded from 61 scalp electrodes using a cap in which $\mathrm{Ag} / \mathrm{AgCl}$ inserts are fixated by individual electrode supports (Easycap System, Falk Minow Services, Munich). All scalp electrodes were referenced to one earlobe during the recording and re-referenced offline to averaged earlobes. Additional electrodes were attached to the outer canthi of both eyes and the sub- and supraorbital ridges of the left or right eye (counterbalanced across participants) for horizontal and vertical EOG recordings. The left or right mastoid (counterbalanced across participants) served as ground. Impedances of all electrodes were kept below $5 \mathrm{k} \Omega$. EEG/ EOG recording and digitization was done by two 32-channel amplifiers (SynAmps, NeuroScan) and NeuroScan software Acquire (sampling rate $=500 \mathrm{~Hz}$ ). DC drift was corrected according to a regression-based algorithm developed by Hennighausen, Heil, and Rösler (1993). Specifically, the vertical EOG channel was filtered with a complex variant of the Haar wavelet. If the magnitude of the resulting signal exceeded a predefined threshold, a blink was detected. The temporal interval in which the magnitude was above threshold was linearly interpolated. The advantage of this procedure over other ocular corrections, which are based mainly on regression analysis, is that it does not alter the amplitude values outside the blink interval, and therefore does not introduce artifacts in the EEG interval that are not affected by eyeblinks. Trials with other artifacts were removed by applying a threshold criterion (maximum voltage range within a trial segment should be lower than $200 \mathrm{~V}$ ).

ERPs were extracted from the edited set of raw data by averaging single trials separately for participants, electrodes, and experimental conditions. Only correct responses on the WM task were used for the ERP average. Furthermore, we excluded the midrange confidence ratings of "probably seen" responses from the analyses in order to minimize the influence of trials with guessing responses. Therefore, analyses of subsequent memory effects contrasted trials with confidently remembered items (i.e., trials with "definitely seen" responses) against trials in which items were forgotten (i.e., trials associated with "probably not seen," "definitely not seen," and, for the first 6 participants, "don't know" responses). As noted earlier, participants with fewer than 15 artifact-free EEG trials in either the remembered or the forgotten condition had to be excluded due to insufficient signal-to-noise ratios. By these criteria, the mean numbers of subsequently remembered and forgotten trials were $32.94(S D=$ $10.13)$ and $28.53(S D=8.61)$ for objects, and $31.64(S D=15.44)$ and $30.14(S D=7.64)$ for letter strings, respectively.

Brain potentials during the WM delay were analyzed by computing mean voltage amplitudes for consecutive 500 -msec intervals beginning with the offset of the stimuli and ending $5 \mathrm{sec}$ later. Poststimulus epochs were baseline corrected relative to the mean amplitude of a 500 -msec epoch preceding cue onset. A subset of 21 electrodes corresponding to the international 10-20 system (Jasper, 1958) entered the statistical analysis (Fp1, Fp2, Fpz, F3, F4, F7, F8, Fz, C3, C4, Cz, T3, T4, T5, T6, P3, P4, Pz, O1, O2, Oz). The statistical analysis followed the hierarchical procedure outlined in Rösler, Friederici, Pütz, and Hahne (1993), in which local effects are tested only if a superordinate analysis signals a significant interaction. First, an overall ANOVA was run for each time epoch. In a second step, the experimental factors were submitted to local $t$ tests for each electrode, provided that the time-point-specific ANOVA had signaled interactions with the factor electrode. This procedure prevents an inflation of Type I error in comparison with an approach that calculates ANOVAs for each electrode-time epoch combination. To further minimize the possibility of Type I errors, only electrodes that revealed significant effects in at least two adjacent time epochs were considered to contribute significantly to the interaction. To account for potential violations of the sphericity assumption, $p$ values were adjusted using the correction of Huynh and Feldt (1976; henceforth, H-F).

\section{RESULTS}

\section{Behavioral Results}

Response times (RTs) and mean percentages of correct responses on the WM task were submitted to ANOVAs with the factors of stimulus type (objects vs. letter strings), delay length ( 5 vs. 6 vs. $7 \mathrm{sec}$ ), and probe type (match vs. nonmatch). To obtain maximum comparability with the corresponding ERP results, this analysis was based on those 12 participants who were also included in the EEG analysis of WM maintenance, independent of memory status. Both ANOVAs revealed significant main effects of stimulus type [for accuracy, $F(1,11)=$ $39.81, p<.0001$; for RT, $F(1,11)=20.72, p<.0001]$, with lower accuracy $(84.7 \%$ vs. $92.8 \%$, respectively) and shorter RTs (913 vs. 1,068 msec, respectively) for objects than for letter strings, suggesting a speed-accuracy tradeoff. No significant effects of delay length [for accuracy, $F(2,11)<1$; for RT, $F(2,11)<1$ ], probe type [for accuracy, $F(1,11)<1$; for RT, $F(1,11)=2.81, p=.1217]$, or interactions (all $F_{\mathrm{S}}<1.88$ ) were observed.

In our next analyses, we examined performance on the subsequent memory test for items associated with correct WM decisions. The distribution of responses across the different response categories for both objects and letter strings is shown in Table 1. In direct correspondence with the EEG analysis of the subsequent memory effects (see below), Figure 2 shows the mean percentages of "definitely seen" judgments for studied and unstudied itemsthat is, the hit and false alarm rates. In order to obtain maximum comparability with the corresponding ERP 
Table 1

Mean Percentages of Responses, by Response Category, for Old and New Objects and Letter Strings

\begin{tabular}{ccccc}
\hline \multirow{2}{*}{$\begin{array}{c}\text { Type of } \\
\text { Stimulus }\end{array}$} & \multicolumn{5}{c}{ Response Category } \\
\cline { 2 - 5 } Objects \\
Old & 43.91 & 20.83 & 15.91 & 17.22 \\
New & 16.43 & 14.78 & 26.43 & 40.26 \\
\multicolumn{5}{c}{ Letter Strings } \\
Old & 30.26 & 28.96 & 24.65 & 13.26 \\
New & 10.08 & 16.87 & 38.17 & 31.13 \\
\hline
\end{tabular}

Note-Old objects are objects that had been presented during the working memory task. 1 , definitely seen; 2 , probably seen; 3 , probably not seen; 4 , definitely not seen.

results, Figure 2 is based on those participants who were also included in the EEG analysis of subsequent memory (16 participants for objects and 14 participants for letter strings). Recognition performance was assessed by comparisons of the hit and false alarm rates. The hit rates were significantly higher than the false alarm rates for both objects $[F(1,15)=169.78, p<.0001]$ and letter strings $[F(1,13)=128.74, p<.0001]$, indicating that subsequent memory performance was well above chance. We also computed $d^{\prime}$ indices with the hit and false alarm rates for both objects and letter strings and ran $t$ tests against zero to find out whether the ability to discriminate between old and new items was significant for objects and for letter strings. This proved to be true for both objects [mean $d^{\prime}=$ $1.16 ; t(15)=12.38, p<.0001]$ and letter strings [mean $\left.d^{\prime}=1.07 ; t(13)=10.38, p<.0001\right]$.

Finally, we ran analyses to determine whether mean RTs differed between subsequently remembered and forgotten objects and letter strings. As above, these analyses included only trials associated with correct decisions

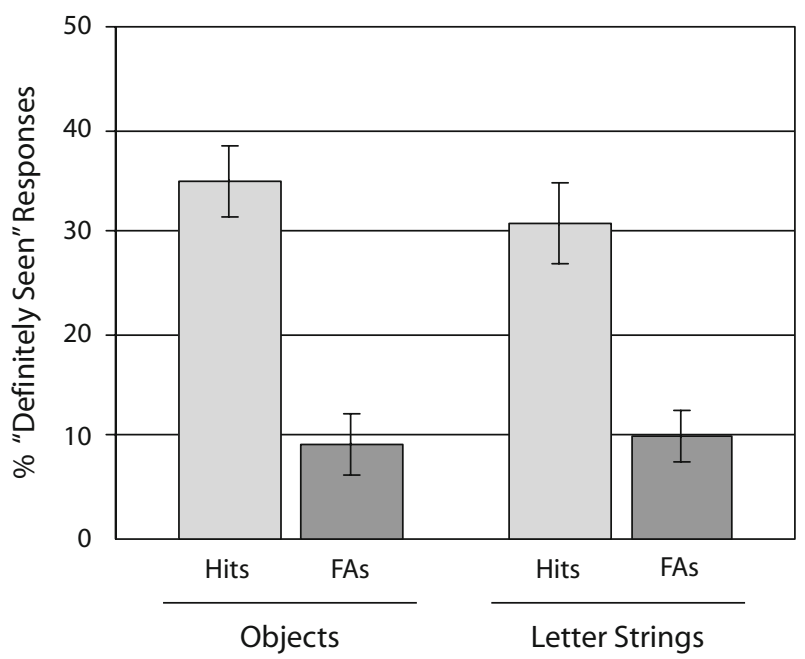

Figure 2. Mean percentages of "definitely seen" judgments for studied and unstudied items (i.e., hit and false alarm [FA] rates). For maximum comparability with the corresponding ERP results, results are shown only for participants who were also included in the corresponding EEG analysis of subsequent memory (16 participants for objects and 14 participants for letter strings). on the corresponding WM trial. No significant RT differences were observed between subsequently remembered and forgotten objects or letter strings [for objects, $\mathrm{RT}($ remembered $)=889 \mathrm{msec}, \mathrm{RT}($ forgotten $)=869 \mathrm{msec}$, $F(1,15)<1$; for letter strings, RT(remembered $)=$ $1,094 \mathrm{msec}$, RT(forgotten) $=1,112 \mathrm{msec}, F(1,13)<1]$.

\section{ERP Data}

The goal of our first analyses was to determine whether slow waves during the WM delay differed as a function of the kind of information that was maintained. As is shown in Figure 3A, independent of memory status, slow negativities were apparent during the WM delay, and the topography of the slow-wave effects differed between objects and letter strings. Slow waves were generally more negative during object maintenance at occipital, parietal, and posterior temporal electrodes, and more negative during letter string maintenance over frontal and anterior temporal electrodes. ANOVAs with factors of stimulus type (objects vs. letter strings) and electrode location were computed for each 500-msec time epoch between 1,000 and 6,000 msec after cue onset (note that the offset of the cue, and thus the start of the delay period, is at $1,000 \mathrm{msec}$ poststimulus). These analyses yielded highly significant stimulus type $X$ electrode interactions for each 500 -msec epoch between 1,000 and 4,000 msec after cue onset [all $F \mathrm{~s}(20,220)>$ 3.88 , all $p \mathrm{~s}<.005]$. Subsequent $t$ tests revealed that, for letter strings, slow waves were significantly more negative over left/middle frontal (Fp1, F7, F3, Fz), left central (C3), and left anterior temporal (T3) sites during WM maintenance; for objects, they were more negative over right occipital $(\mathrm{O} 2)$, parietal $(\mathrm{P} 4)$, and posterior temporal (T6) sites during WM maintenance (see Figure 3B). To test whether these differences remain reliable if mean and variance differences between the experimental conditions are eliminated, amplitude measures were averaged across the 1,000- to 4,000-msec epoch and $z$ standardized for each subject and experimental condition. The interaction remained significant also after $z$ standardization for the 21 electrodes $[F(20,220)=3.11, p=.0026, \varepsilon(\mathrm{H}-\mathrm{F})=$ $.445]$, as well as for all 61 electrodes $[F(60,660)=2.97$, $p=.0032, \varepsilon(\mathrm{H}-\mathrm{F})=.155]$, taking into account the complete available topographical information.

Our next set of analyses addressed the relationship between activity during the WM delay (i.e., the 1,000 to $6,000,7,000$, or $8,000 \mathrm{msec}$ following onset of the cue stimulus - see Figure 1) and subsequent LTM performance. Figures 4 and 5 depict the ERPs for remembered and forgotten objects and letter strings, respectively, showing that slow-wave amplitudes during the delay period clearly differed with respect to LTM performance. For objects, the difference between remembered and forgotten stimuli was most pronounced at occipital and posterior temporal electrodes; for letter strings, the difference was greatest over left frontal and anterior temporal electrodes, with a maximum difference at the left frontal electrode F7. ANOVAs with LTM performance (remembered vs. forgotten) and electrode location as factors were run separately for each stimulus type and each time epoch. In the analyses of object trials, we observed 

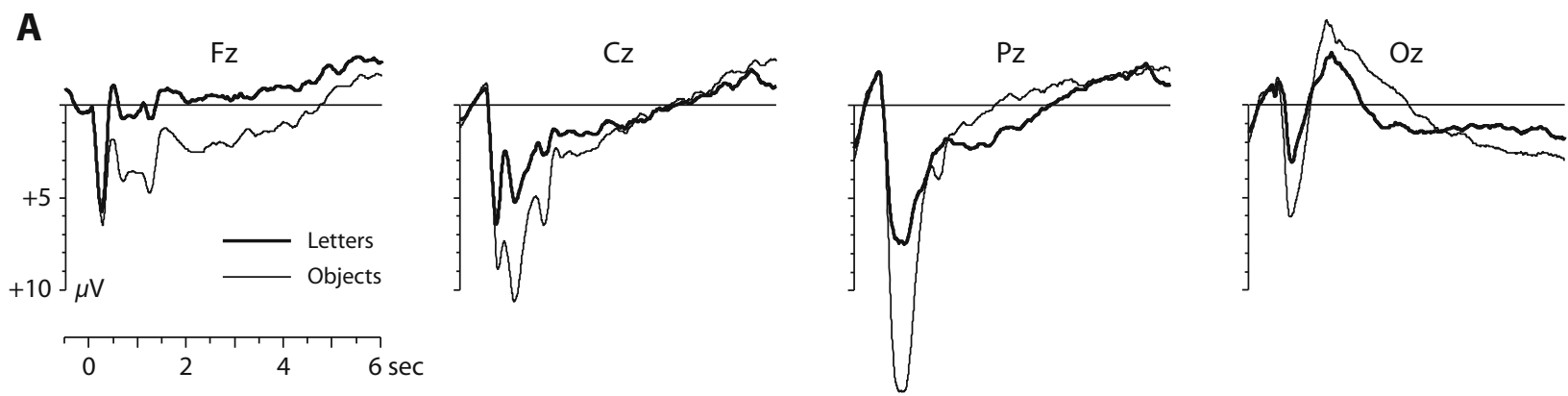

B
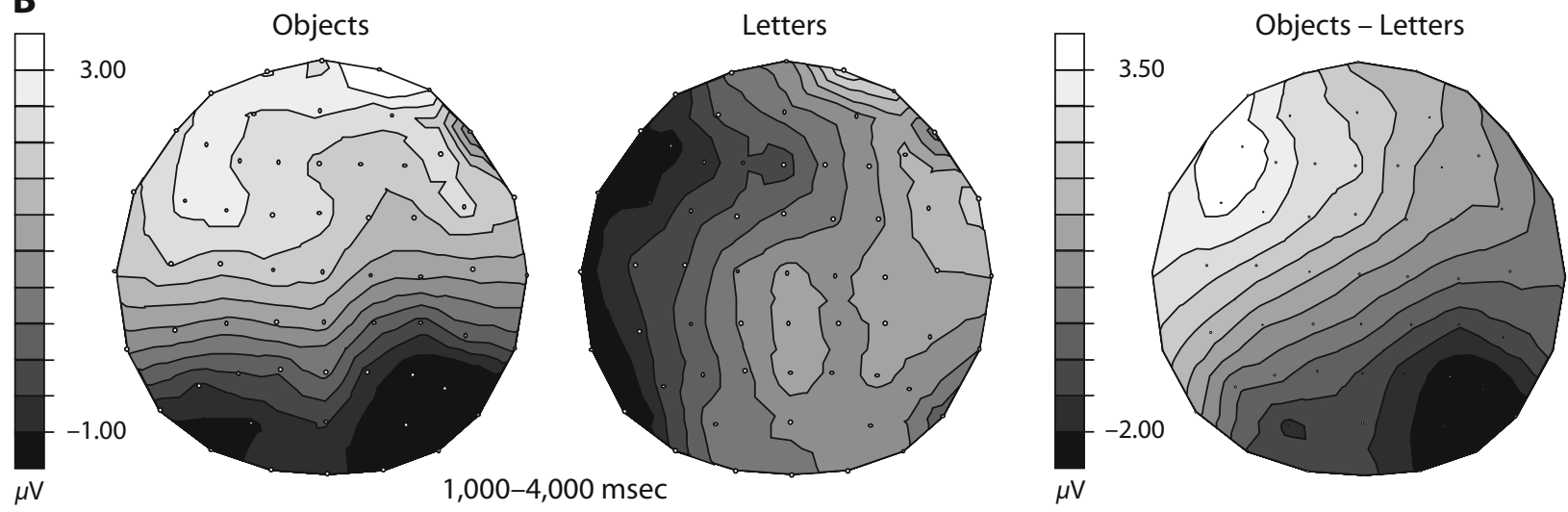

Figure 3. Slow waves during WM maintenance exhibit material-specific topographies. (A) ERPs for objects and letter strings, independent of subsequent memory performance. Slow-wave amplitudes during the delay period were generally more negative during object maintenance at occipital, parietal, and posterior temporal electrodes, and more negative during letter string maintenance over frontal and anterior temporal electrodes. (Note that in this panel and in Figures 4, 5, and 7, negativity is plotted upward.) (B) Topographic maps of ERP amplitudes during maintenance of objects and letter strings. On the right side is the map of the difference between object maintenance and letter string maintenance. Topographic maps in this figure and in Figure 6 are based on data from all 61 scalp electrodes. More negative amplitudes are coded by darker shading.

significant LTM performance $\times$ electrode interactions $1,000-2,500$ msec after cue onset $\left[F \_\max (20,300)=\right.$ $4.08, \varepsilon(\mathrm{H}-\mathrm{F})=.280, p=.0016 ; F \_\min (20,300)=2.45$, $\varepsilon(\mathrm{H}-\mathrm{F})=.271, p=.0361]$. (The terms $F \_$max and $F \_$min refer to the largest and smallest $F$ values, respectively, of all contiguous time epochs that show a significant interaction.) Electrode-wise $t$ tests showed that slow waves were significantly more negative for remembered than for forgotten objects at occipital $(\mathrm{O} 1, \mathrm{Oz})$ and posterior temporal (T5) electrodes. On letter string trials, we observed significant LTM performance $\times$ electrode interactions $1,500-4,500 \mathrm{msec}$ after cue onset $\left[F \_\max (20,260)=\right.$ $4.04, \varepsilon(\mathrm{H}-\mathrm{F})=.243, p=.0033 ; F \_\min (20,260)=2.50$, $\varepsilon(\mathrm{H}-\mathrm{F})=.248, p=.0400]$. Electrode-wise $t$ tests showed that slow waves were significantly more negative for remembered than for forgotten letter strings at left frontal electrode F7, whereas potentials were more positive for remembered letter strings at $\mathrm{Fp} 2$ and Fpz.

To directly test the assumption that the subsequent memory effects are significant only from 1,000 to $2,500 \mathrm{msec}$ for objects and from 1,500 to $4,500 \mathrm{msec}$ for letter strings, but not later during the delay, material-specific ANOVAs with factors of time, electrode, and experimental condition (remembered vs. forgotten) were computed, revealing three-way interactions for letter strings $[F(20,260)=$ $3.05, p=.0217, \varepsilon(\mathrm{H}-\mathrm{F})=.215]$ and objects $[F(20,300)=$
$2.19, p=.0566, \varepsilon(\mathrm{H}-\mathrm{F})=.292]$. As was expected, post hoc tests revealed significant electrode $\times$ experimental condition interactions only during the first time window for both objects [for 1,000-2,500 msec, $F(20,300)=$ $4.03, p=.0017, \varepsilon(\mathrm{H}-\mathrm{F})=.280$; for $2,500-6,000 \mathrm{msec}$, $F(20,300)=1.00, p=.4215, \varepsilon(\mathrm{H}-\mathrm{F})=.248]$ and letter strings [for 1,500-4,500 msec, $F(20,300)=3.56, p=$ $.0097, \varepsilon(\mathrm{H}-\mathrm{F})=.218$; for $4,500-6,000 \mathrm{msec}, F(20,300)=$ $1.55, p=.1947, \varepsilon(\mathrm{H}-\mathrm{F})=.225]$.

The topographical maps for remembered and forgotten objects and letter strings, as well as their difference maps, are shown in Figure 6. The amplitudes of slow potentials during maintenance of remembered (Figure 6A, left) and forgotten (Figure 6A, middle) objects were most negative over occipital scalp sites, whereas the most negative potentials for both remembered (Figure 6B, left) and forgotten (Figure 6B, middle) letter strings were found over the left frontal cortex. A similar pattern was evident in topographic maps of ERP differences between remembered and forgotten objects (Figure 6A, right) and letter strings (Figure 6B, right), with the most pronounced differences for objects apparent over the occipital cortex and those for letter strings more broadly distributed across sites over the left frontal cortex.

Figure 7 shows ERP amplitudes for subsequently remembered (hits) and forgotten (misses) objects and letter 

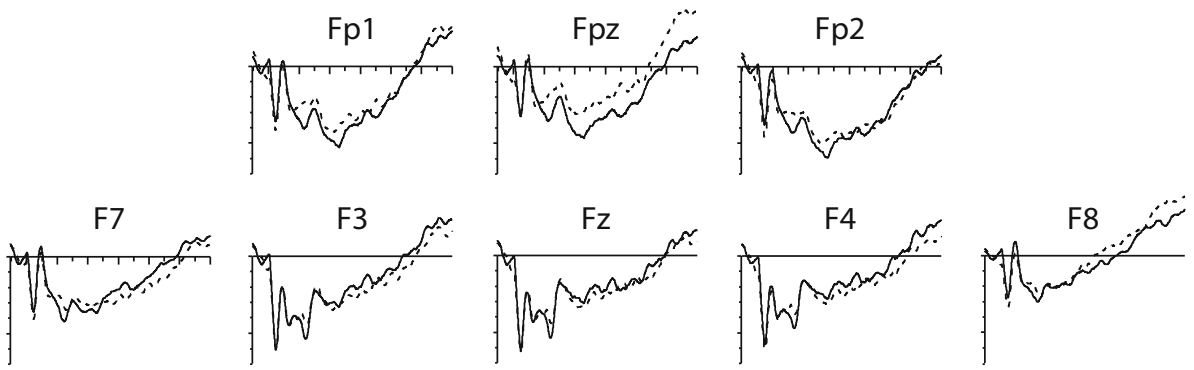

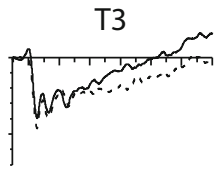

T5

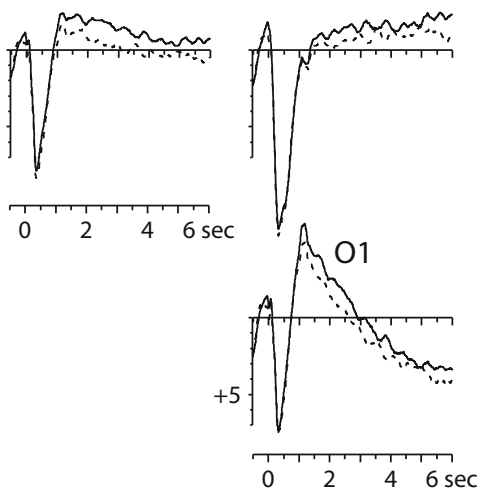

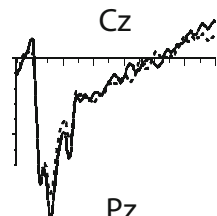

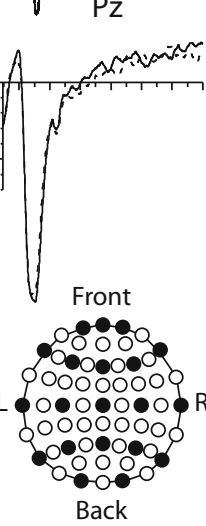

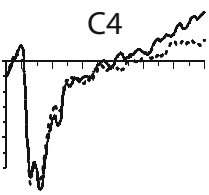

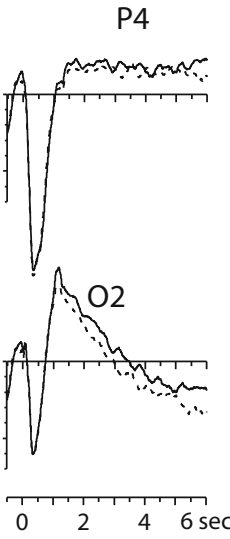

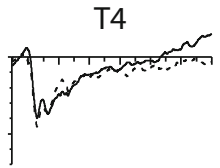

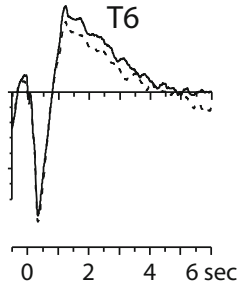

Objects

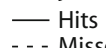

- - Misses

Figure 4. ERPs for remembered and forgotten objects (hits and misses, respectively), showing that the slow-wave amplitudes during the WM delay period differed with respect to LTM performance. Slow potentials were more negative for remembered than for forgotten stimuli at occipital and posterior temporal electrodes. Locations of plotted electrodes are schematically depicted as the filled circles in the small inset at the bottom of the figure. strings at electrodes $\mathrm{O} 1$ and F7 for the time windows in which significant LTM performance $\times$ electrode interactions were found in the statistical analyses. Negative slow waves emerged at $\mathrm{O} 1$ only when objects had to be remembered, and at F7 only if letter strings had to be remembered. Furthermore, the difference between remembered and forgotten items reached statistical significance at O1 only for objects, and at F7 only for letter strings, which was revealed by specific comparisons of the timeaveraged ERP amplitudes for remembered versus forgotten objects [for O1, $F(1,15)=7.61, p=.0146$; for F7, $F(1,15)=2.41, p=.1413$ ] and letter strings [for $\mathrm{O} 1$, $F(1,13)=1.31, p=.2737$; for $\mathrm{F} 7, F(1,13)=7.64, p=$ $.0161]$. Because different but overlapping groups of participants entered the statistical analyses for objects and letter strings, it was not possible to compute an ANOVA with factors of stimulus type (objects vs. letter strings), electrode location (O1 vs. F7), and memory status (remembered vs. forgotten) on the data presented in Figure 5 in order to substantiate the asymmetry between F7 and $\mathrm{O} 1$ by means of a significant three-way interaction. However, we computed this ANOVA with a reduced sample of those 12 participants who were included in both the objects and the letter strings analyses and found a marginally signifi- cant three-way interaction $[F(1,11)=4.20, p=.0651]$. Specific comparisons revealed that the difference between remembered and forgotten items reached statistical significance at $\mathrm{O} 1$ only for objects [for O1, $F(1,11)=5.86$, $p=.0339$; for F7, $F(1,11)<1]$ and at F7 only for letter strings [for $\mathrm{O} 1, F(1,13)<1$; for $\mathrm{F} 7, F(1,11)=8.18, p=$ .0060].

The preceding analyses characterized ERPs during the memory delay, which presumably reflected WM maintenance processes. A final set of analyses was aimed at detecting possible ERP differences related to stimulus encoding (i.e., processing of the cue stimulus). This analysis was critical to confirm that subsequent memory effects during the delay could not be attributed to encoding-related effects. Accordingly, ANOVAs were computed for consecutive time epochs of $50 \mathrm{msec}$, ranging from the onset to the offset of the cue (i.e., from 0 to $1,000 \mathrm{msec}$ poststimulus). Here, we found no evidence for differences based on LTM performance (i.e., no main effects of LTM performance or LTM performance $\times$ electrode interactions) for either objects or letter strings. However, in a comparison of objects and letter strings independent of LTM performance, a stimulus type $\times$ electrode interaction was evident between 100 and 

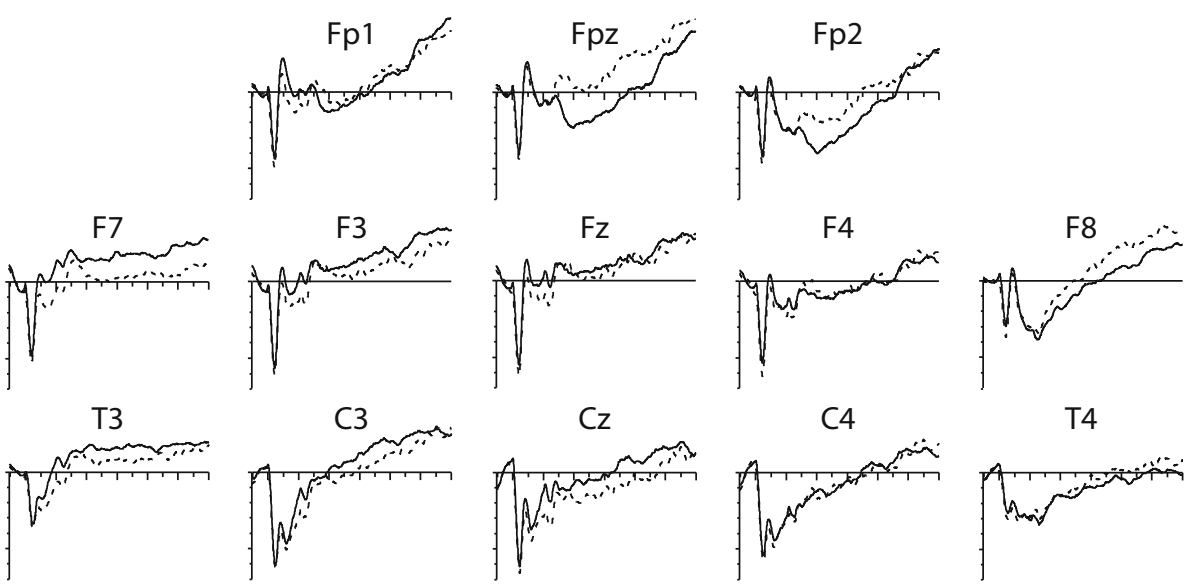

T4
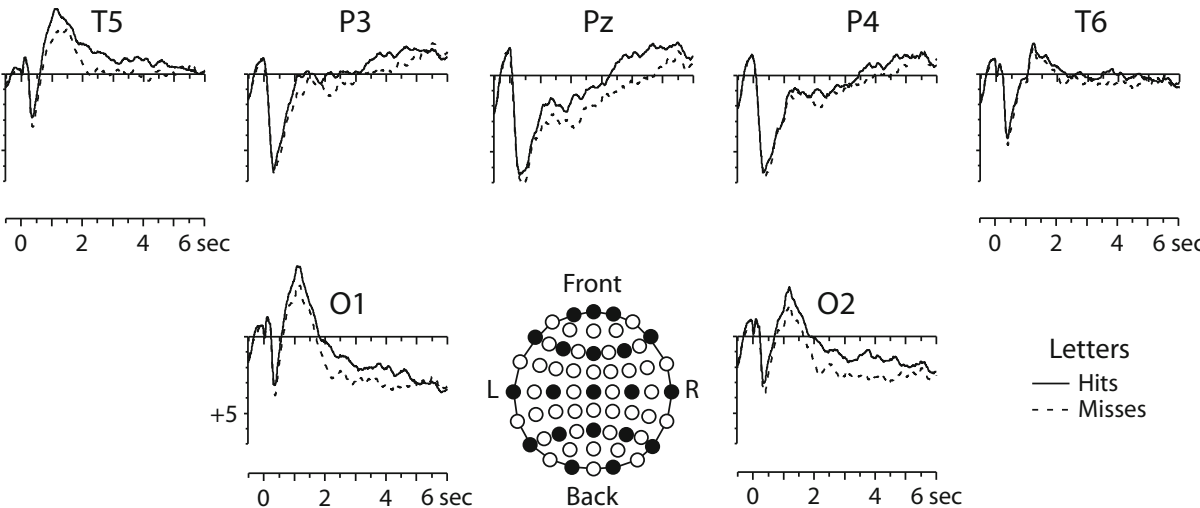

Figure 5. ERPs for remembered and forgotten letter strings (hits and misses, respectively), showing that slow-wave amplitudes during the delay period differed with respect to LTM performance. Slow potentials were more negative for remembered than for forgotten stimuli over left frontal and anterior temporal electrodes. Locations of plotted electrodes are schematically depicted as the filled circles in the small inset at the bottom of the figure.

$1,000 \mathrm{msec}$ after cue onset $\left[F \_\max (20,460)=32.10\right.$, $p<.0001, \varepsilon(\mathrm{H}-\mathrm{F})=.179 ; F_{-} \min (20,460)=5.67$, $p=.0025, \varepsilon(\mathrm{H}-\mathrm{F})=.134]$, showing that ERPs were generally more positive when objects were presented. Electrode-wise $t$ tests proved that this effect was broadly distributed over occipital, parietal, central, temporal, and frontal areas, with a maximum of around $500 \mathrm{msec}$ at electrode Pz (see Figure 3A).

\section{DISCUSSION}

In the present study, we investigated the relationship between WM maintenance and successful LTM formation using visually presented objects and letter strings as stimuli. Slow ERPs over the parietal and occipital cortex were more negative during WM maintenance of objects, and over the left frontal cortex during WM maintenance of letter strings. Furthermore, they were generally more negative for items that were subsequently successfully remembered than for subsequently forgotten items. Subsequent memory effects associated with objects and letter strings were topographically distinct, with maximum effects at those electrodes that showed the maximum negativity during maintenance.
The present results are directly relevant to debates regarding the relationship between WM maintenance and LTM formation. As noted earlier, some researchers have suggested that WM maintenance serves to strengthen LTM traces (Atkinson \& Shiffrin, 1968; Hebb, 1949), whereas others have argued that maintenance, in the absence of elaboration, does not contribute to successful LTM formation (Craik \& Watkins, 1973). In principle, our participants could have been using elaboration strategies during the delay interval. However, several arguments speak against this possibility. First, the response after the delay had to be given on a purely perceptual basis. Thus, "deep" semantic elaboration did not help to improve performance. Furthermore, all stimuli were meaningless, which makes semantic elaborations unlikely. Finally, the participants did not know that a recognition test would follow and thus had no motivation to store the material for longer than the few seconds of the delay, and the participants' reports at the end of the experiment confirmed that they had not tried to elaborate the stimuli. Therefore, our results are more consistent with the view that rote WM maintenance serves to strengthen LTM traces.

In the light of evidence demonstrating that slow-wave negativity reflects neural activation of the underlying 


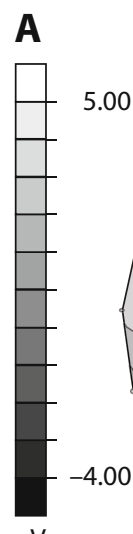

$\mu \mathrm{V}$

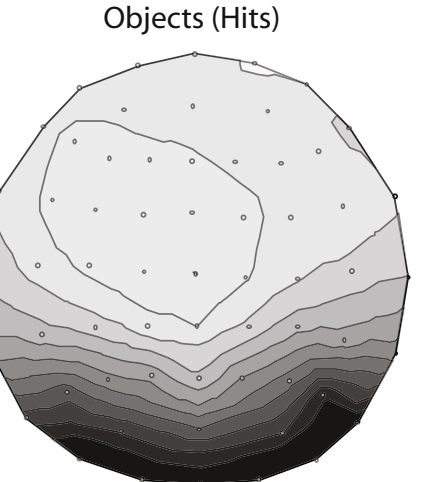

$1,000-2,500 \mathrm{msec}$

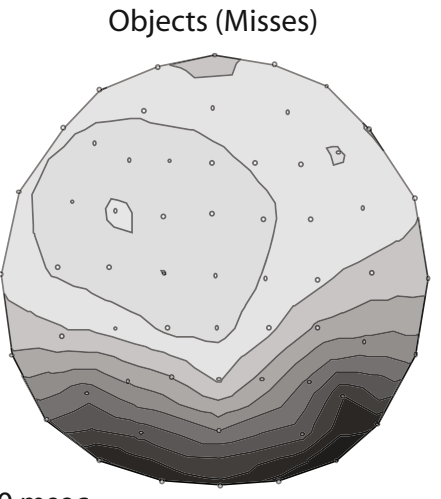

B

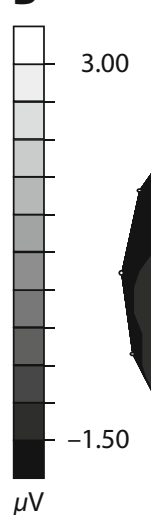

Letters (Hits)

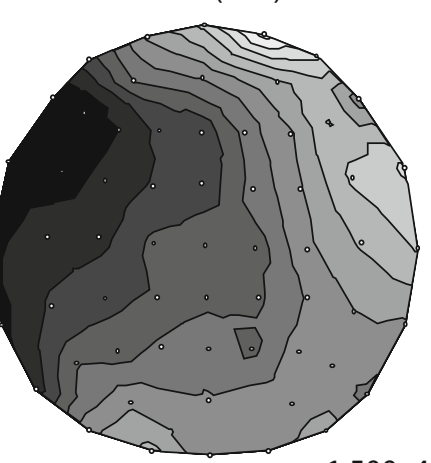

$1,500-4,500 \mathrm{msec}$

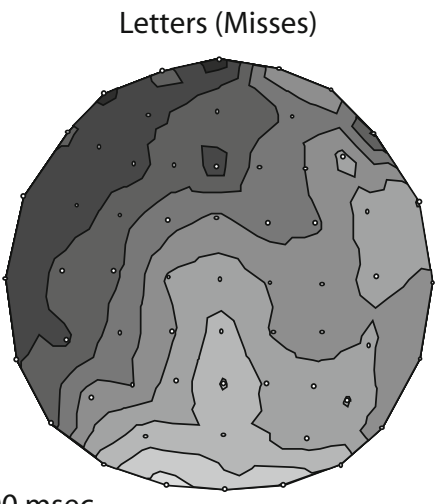

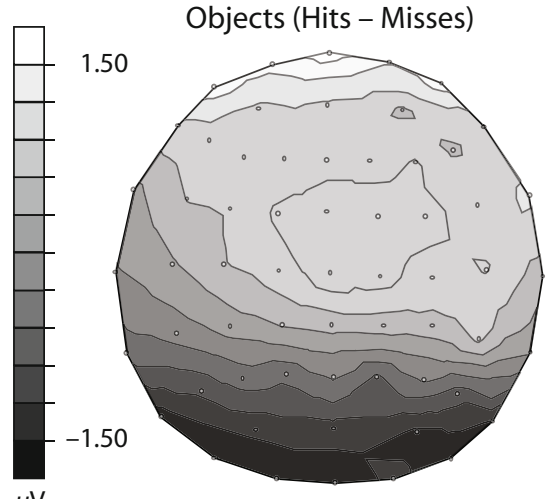

$\mu \mathrm{V}$

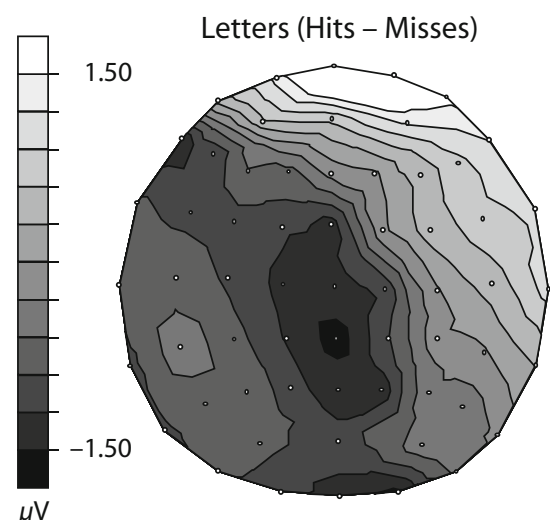

Figure 6. Topographic maps of slow-wave amplitudes for (left) remembered and (middle) forgotten stimuli (hits and misses, respectively), as well as the remembered - forgotten difference (right). (A) Maps for objects. (B) Maps for letter strings. Each map depicts mean ERP amplitudes during the time intervals with significant interactions between memory performance and electrode location.

cortical tissue (Elbert, 1993; Khader, Schicke, Röder, \& Rösler, in press; McCallum \& Curry, 1993; Mitzdorf, 1985; Speckmann, Caspers, \& Elger, 1984), we propose that, in the present study, neural activity recorded during WM maintenance was enhanced for items that were later remembered relative to items that were later forgotten. Furthermore, this enhanced activity was probably generated in brain areas that mediate the maintenance of specific kinds of information in WM. Therefore, the results are consistent with the idea that maintenance of information in material-specific cortical networks serves to strengthen LTM traces in those networks (Hebb, 1949).

The conclusions above rest on the assumption that slow potentials emerging during the delay are related to WM maintenance. This assumption is supported by several lines of evidence. Most importantly, the topography of slow waves during the delay differed depending on whether verbal or visuospatial information had to be maintained. This finding is consistent with behavioral studies that demonstrated qualitatively different mechanisms for verbal and for visual object information (see Baddeley, 1986, 1992, for reviews). Furthermore, the peak negativities for verbal and for visual object information are located over cortical areas that have been shown to be active during maintenance of these kinds of materials (e.g., Jonides et al., 2003; Smith \& Jonides, 1997).

Although present methods do not allow for precise localization of the cortical regions that generate scalp-recorded ERPs (due to volume conduction and variability in the orientation of generating dipoles; Nunez, 1981), there is evidence that slow negative brain potentials originate from cortical field potentials in close proximity to the recording electrode (McCallum \& Curry, 1993; Mitzdorf, 1985, 1991; Speckmann et al., 1984). Accordingly, the topographies of slow potentials observed in the present study are consistent with the idea that object maintenance, and its effect on LTM formation, may emerge through activation of networks in the occipital cortex. In the case of letter strings, these processes may reflect activation of networks in the left frontal cortex. These speculations are bolstered by converging evidence from neuroimaging studies showing that visual WM maintenance elicits activity in the occipital cortex, whereas verbal WM maintenance activates the left frontal cortex (Jonides et al., 2003; Smith \& Jonides, 1997; Smith, Jonides, \& Koeppe, 1996). Furthermore, neuroimaging studies have reported subsequent memory effects in the occipital cortex during visual WM maintenance (Ranganath et al., 2005; Schon et al., 2004) and in the left infe- 


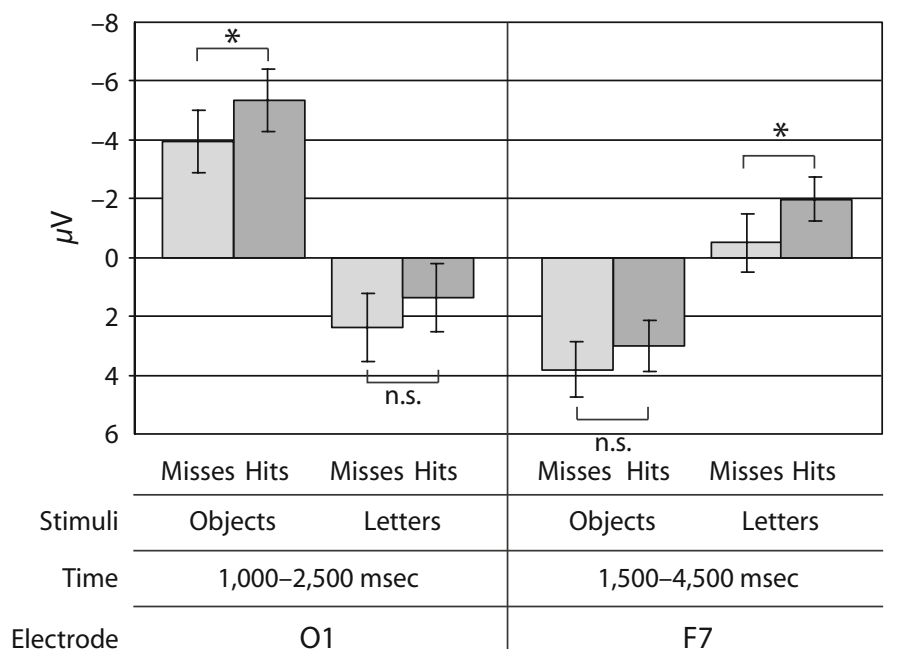

Figure 7. Mean ERP amplitudes for subsequently remembered and forgotten objects (hits and misses, respectively) and letter strings at electrodes $\mathrm{O} 1$ and $\mathrm{F} 7$ for those time windows in which significant interactions between memory performance and electrode location were observed (see the Method section). Error bars depict the standard errors of the mean. "Significant differences $(p<.05)$.

rior prefrontal cortex during verbal WM maintenance (Blumenfeld \& Ranganath, 2006; Davachi et al., 2001; Köhler, Paus, Buckner, \& Milner, 2004; Wagner et al., 1998).

The assumption that slow waves emerging during a WM delay are functionally related to WM maintenance is also strongly supported by a previous study (Rolke, Heil, Hennighausen, Häussler, \& Rösler, 2000) that showed a direct relationship between memory load and slow-wave amplitude during a WM task. In that study, slow potentials were recorded while subjects had to mentally transform the order of sequentially presented words (verbal condition) or positions within a grid (spatial condition). The difficulty of the transformation process - that is, the amount of load imposed on WM resources - was varied systematically. The amplitudes of the slow waves elicited during the transformation process were found to vary with task difficulty. Most importantly, and in consistency with the results of the present study, the topography of this amplitude modulation differed between the verbal and the spatial conditions, with the maximum effect for verbal information over the left frontal cortex and that for spatial information over the parietal cortex. These results strongly suggest that slow waves during the delay reflect the maintenance of representations in specific cortical areas (see Ruchkin, Grafman, Cameron, \& Berndt, 2003, for further evidence from slow ERPs that information-specific cortical areas are recruited in the maintenance and manipulation of WM contents).

Analyses of the temporal dynamics of the subsequent memory effects revealed significant LTM performance $X$ electrode interactions only during the early phase of the maintenance epoch for objects, and during the early and middle maintenance epochs for letter strings. These results suggest that, at least for objects, processing during the initial part of the delay was predictive of LTM perfor- mance and are, therefore, in accordance with other recent behavioral and neuroimaging results (Johnson et al., 2002; Naveh-Benjamin \& Jonides, 1984a, 1984b; Ranganath et al., 2005), showing that processing during the initial stage of WM maintenance disproportionately promotes effective LTM formation. This effect is explained by assuming that, in the first stage, recently encoded sensory stimuli are transformed into representational (e.g., articulatory, phonological, visual, oculomotor) codes, which can be maintained in the absence of external stimulation. Once this code is established, a second, more automated stage of rehearsal can proceed. However, it is unlikely that these stages can be associated with specific time windows, because the duration of processing would be expected to differ under different conditions. For example, it is likely that the early stage would be much longer for complex, unfamiliar stimuli (e.g., 1-3 sec) than for simple, familiar stimuli (e.g., 500-1,000 msec).

Although the ERP results in this study do not suggest a specific time course for the early and late stages of WM maintenance, they substantiate the general distinction between two separate stages. Therefore, our results are consistent with the notions that WM maintenance is no unitary process (Naveh-Benjamin \& Jonides, 1984b; Ranganath et al., 2005) and that the engagement of executive control during the initial stage of WM maintenance contributes to LTM formation (Johnson et al., 2002; NavehBenjamin \& Jonides, 1984b; Ranganath et al., 2005). However, the comparisons of ERP results between object and letter string trials are not entirely consistent with this hypothesis. If the relationship between WM maintenance and LTM were mediated solely by the engagement of a nonspecific control process, one would expect to observe similar subsequent memory effects for both categories of stimuli. The fact that the largest effects were observed at 
the electrodes that showed the maximum negativity during maintenance in general (i.e., F7 for letter strings and O1 for objects) suggests that the effect of maintenance on LTM formation may be mediated by the same materialspecific networks that are involved in WM maintenance in general. We therefore suggest that the relationship between WM maintenance and LTM is not mediated solely by the effortful engagement of executive control, but that it may emerge through an interaction between control processes and representations of the information that are being formed and maintained. This view corresponds well with models that see WM maintenance as an emergent property of interactions between regions involved in executive control and regions that represent information that has to be maintained (Postle, 2006; Ranganath, 2006; Ranganath \& D'Esposito, 2005).

The slow-wave subsequent memory effect evoked by objects had a more phasic time course than that evoked by letter strings. The object effect was reliable at $\mathrm{O} 1$ between 1,000 and 2,500 msec, whereas the letter effect was significant at $\mathrm{F} 7$ during almost the entire delay period - that is, between 1,500 and 4,500 msec. These different time courses are consistent with the idea that different processes are recruited to maintain different kinds of materials. For example, Baddeley $(1986,1992)$ suggested that letter strings are maintained in the "phonological loop" through serial verbal rehearsal. Objects, on the other hand, are maintained in the "visuospatial sketchpad" as analogue visual representations through visual imagery (Logie, 1995). Assuming that the slow waves during the delay period are a correlate of WM maintenance processes, the distinct time courses are potentially compatible with Baddeley's predictions: Serial rehearsal of letters may continue during the whole epoch, whereas maintenance of a holistic image may only require one to "refresh" (Johnson et al., 2002) the representation of the stimulus at the beginning of the delay. That the two types of stimuli are handled differently in WM is also substantiated by our participants' debriefings. Participants reported using serial rehearsal to maintain letter strings. In contrast, for objects, they reported forming a mental image at the beginning of the delay, which became less vivid over the course of the delay period. A more objective piece of evidence comes from Ranganath et al. (2005), who conducted a behavioral study using the same objects that were used in the present study. Their results showed that presentation of visual distractors during the WM delay affected subsequent LTM performance only if the distractors appeared shortly $(1 \mathrm{sec})$ after the onset of the delay period. Presenting the distractors later in the delay (e.g., 4 or $7 \mathrm{sec}$ after the onset of the delay) had no effect on subsequent memory.

In contrast to many findings of more positive ERPs for subsequently remembered items than for subsequently forgotten items (i.e., the difference due to memory effect; for reviews, see, e.g., Paller, Kutas, \& Mayes, 1987; Paller, McCarthy, \& Wood, 1988; see Paller \& Wagner, 2002; Rugg, 1995), in the present study we found more negative potentials for subsequently remembered items. This finding is not surprising, in that the subsequent memory effects reported here reflected modulations of slow DC poten- tials. As noted above, DC potentials have been associated with temporally extended WM processes, with increasing negativity reflecting increasing activation. Accordingly, it makes sense that enhanced negativity early in the delay (presumably a correlate of more extensive WM processing) was correlated with successful LTM formation.

Nonetheless, one might wonder why a positive ERP modulation was not observed during stimulus encoding, as has been observed in other studies. A possible explanation of this result is provided by a recent study by Otten, Sveen, and Quayle (2006), which demonstrated that distinct patterns of electrical brain activity occurred during memory formation of novel items (nonwords) versus familiar items (words). Whereas the ERP subsequent memory effect for words showed the classical pattern of more positive-going potentials for remembered items from around $500 \mathrm{msec}$ after word onset, the nonword ERP subsequent memory effect was more negative-going for remembered items from around $1 \mathrm{sec}$ onward. These results suggest that the brain supports the encoding of novel versus familiar information in qualitatively different ways, including the engagement of distinct neural activity at different points in time (Otten et al., 2006).

In conclusion, the results from the present study are strongly consistent with the idea that cognitive and neural processing during WM maintenance promotes successful LTM formation, and that this effect is likely to be mediated by the strengthening of stimulus-specific memory traces.

\section{AUTHOR NOTE}

This work was supported by Grant FOR254/2-1 of the German Research Foundation (DFG) assigned to F.R. and by Public Health Service Grant 1R01MH068721 from the National Institute of Mental Health to C.R. We thank Erwin Hennighausen for technical assistance and Kerstin Jost for valuable comments and helpful discussions. Correspondence concerning this article should be addressed to P. Khader, Department of Psychology, Philipps University Marburg, 35032 Marburg, Germany (e-mail: khader@staff.uni-marburg.de; Web site, staff-www.uni-marburg.de/ cablab).

\section{REFERENCES}

Arthurs, O. J., \& Boniface, S. (2002). How well do we understand the neural origins of the fMRI BOLD signal? Trends in Neurosciences, 25, 27-31.

Atkinson, R. C., \& Shiffrin, R. M. (1968). Human memory: A proposed system and its control processes. In K. W. Spence \& J. T. Spence (Eds.), The psychology of learning and motivation (Vol. 2, pp. 89105). New York: Academic Press.

BADDELEY, A. (1986). Working memory. Oxford: Oxford University Press. Baddeley, A. (1992). Working memory. Science, 255, 556-559.

Birbaumer, N., Elbert, T., Canavan, A. G., \& Rockstroh, B. (1990). Slow potentials of the cerebral cortex and behavior. Physiological Reviews, 70, 1-41.

Blumenfeld, R. S., \& Ranganath, C. (2006). Dorsolateral prefrontal cortex promotes long-term memory formation through its role in working memory organization. Journal of Neuroscience, 26, 916925.

Craik, F. I. M. (2002). Levels of processing: Past, present . . . and future? Memory, 10, 305-318.

Craik, F. I. M., \& Lockhart, R. S. (1972). Levels of processing: A framework for memory research. Journal of Verbal Learning \& Verbal Behavior, 11, 671-684.

CRAIK, F. I. M., \& WATKINS, M. J. (1973). The role of rehearsal in shortterm memory. Journal of Verbal Learning \& Verbal Behavior, 12, 599-607. 
DaVachi, L., Maril, A., \& Wagner, A. D. (2001). When keeping in mind supports later bringing to mind: Neural markers of phonological rehearsal predict subsequent remembering. Journal of Cognitive Neuroscience, 13, 1059-1070.

ELBERT, T. (1993). Slow cortical potentials reflect the regulation of cortical excitability. In W. C. McCallum \& S. H. Curry (Eds.), Slow potential changes in the human brain (pp. 235-251). New York: Plenum.

FusteR, J. M. (1995). Memory in the cerebral cortex: An empirical approach to neural networks in the human and nonhuman primate. Cambridge, MA: MIT Press.

HebB, D. O. (1949). The organization of behavior: A neuropsychological theory. New York: Wiley.

Hennighausen, E., Heil, M., \& Rösler, F. (1993). A correction method for DC drift artifacts. Electroencephalography \& Clinical Neurophysiology, 86, 199-204.

HuYNH, H., \& FELDT, L. S. (1976). Estimation of the Box correction for degrees of freedom from sample data in randomized block and splitplot designs. Journal of Educational Statistics, 1, 69-82.

JASPER, H. (1958). The ten-twenty electrode system of the Internationa Federation. Electroencephalography \& Clinical Neurophysiology, 10, 371-375.

Johnson, M. K., Reeder, J. A., Raye, C. L., \& Mitchell, K. J. (2002). Second thoughts versus second looks: An age-related deficit in reflectively refreshing just-activated information. Psychological Science, 13, 64-67.

Joliceur, P., \& Dell'Acqua, R. (1998). The demonstration of shortterm consolidation. Cognitive Psychology, 36, 138-202.

Jonides, J., Sylvester, C.-Y. C., Lacey, S. C., Wager, T. D., NichOLs, T. E., \& AwH, E. (2003). Modules of working memory. In R. H. Kluwe, G. Lüer, \& F. Rösler (Eds.), Principles of learning and memory (pp. 113-134). Cambridge, MA: Birkhäuser.

Khader, P., Schicke, T., RöDer, B., \& Rösler, F. (in press). Evidence for a close correspondence of DC-recorded slow ERPs and BOLD signal changes in humans. International Journal of Psychophysiology.

KöHler, S., Paus, T., Buckner, R. L., \& Milner, B. (2004). Effects of left inferior prefrontal stimulation on episodic memory formation: A two-stage fMRI-rTMS study. Journal of Cognitive Neuroscience, 16, 178-188.

LogIE, R. H. (1995). Visuo-spatial working memory. Hove, U.K.: Erlbaum.

Logothetis, N. K., Pauls, J., Augath, M., Trinath, T., \& OelterMANN, A. (2001). Neurophysiological investigation of the basis of the fMRI signal. Nature, 412, 150-157.

McCallum, W. C., \& CurRy, S. H. (1993). Slow potential changes in the human brain. New York: Plenum.

MitzDORF, U. (1985). Current source-density method and application in cat cerebral cortex: Investigation of evoked potentials and EEG phenomena. Physiological Reviews, 65, 37-100.

MitzDorf, U. (1991). Physiological sources of evoked potentials. In C. H. M. Brunia, G. Mulder, \& M. N. Verbaten (Eds.), Event-related brain research (pp. 47-57). Amsterdam: Elsevier.

Mukamel, R., Gelbard, H., Arieli, A., Hasson, U., Fried, I., \& Mal$\mathrm{ACH}, \mathrm{R}$. (2005). Coupling between neuronal firing, field potentials, and fMRI in human auditory cortex. Science, 309, 951-954.

Naveh-Benjamin, M., \& Jonides, J. (1984a). Cognitive load and maintenance rehearsal. Journal of Verbal Learning \& Verbal Behavior, 23, 494-507.

Naveh-Benjamin, M., \& Jonides, J. (1984b). Maintenance rehearsal: A two-component analysis. Journal of Experimental Psychology: Learning, Memory, \& Cognition, 10, 369-385.

Nunez, P. L. (1981). Electrical fields of the brain. New York: Oxford University Press.

OLDFIELD, R. C. (1971). The assessment and analysis of handedness: The Edinburgh inventory. Neuropsychologia, 9, 97-113.

Otten, L., Sveen, J., \& Quayle, A. (2006). The roles of meaning and novelty in memory formation: An event-related potential study [Abstract]. Journal of Cognitive Neuroscience, 18(Suppl.), 228.

Paller, K. A., Kutas, M., \& Mayes, A. R. (1987). Neural correlates of encoding in an incidental learning paradigm. Electroencephalography \& Clinical Neurophysiology, 67, 360-371.

Paller, K. A., McCarthy, G., \& Wood, C. C. (1988). ERPs predictive of subsequent recall and recognition performance. Biological Psychology, 26, 269-276.
Paller, K. A., \& WaGner, A. D. (2002). Observing the transformation of experience into memory. Trends in Cognitive Sciences, 6, 93-102.

Pasternak, T., \& Greenlee, M. W. (2005). Working memory in primate sensory systems. Nature Reviews Neuroscience, 6, 97-107.

PostLe, B. R. (2006). Working memory as an emergent property of the mind and brain. Neuroscience, 139, 23-38.

Ranganath, C. (2006). Working memory for visual objects: Complementary roles of inferior temporal, medial temporal, and prefrontal cortex. Neuroscience, 139, 277-289.

Ranganath, C., Cohen, M. X., \& Brozinsky, C. J. (2005). Working memory maintenance contributes to long-term memory formation: Neural and behavioral evidence. Journal of Cognitive Neuroscience, 17, 994-1010.

Ranganath, C., \& D'Esposito, M. (2005). Directing the mind's eye: Prefrontal, inferior and medial temporal mechanisms for visual working memory. Current Opinion in Neurobiology, 15, 175-182.

Raye, C. L., Johnson, M. K., Mitchell, K. J., Reeder, J. A., \& Greene, E. J. (2002). Neuroimaging a single thought: Dorsolateral PFC activity associated with refreshing just-activated information. Neurolmage, 15, 447-453.

Rolke, B., Heil, M., Hennighausen, E., Häussler, C., \& Rösler, F. (2000). Topography of brain electrical activity dissociates the sequential order transformation of verbal versus spatial information in humans. Neuroscience Letters, 282, 81-84.

Rösler, F., Friederici, A. D., Pütz, P., \& Hahne, A. (1993). Eventrelated brain potentials while encountering semantic and syntactic constraint violations. Journal of Cognitive Neuroscience, 5, 345-362.

RöSLER, F., HEIL, M., \& RöDER, B. (1997). Slow negative brain potentials as reflections of specific modular resources of cognition. Biological Psychology, 45, 109-141.

Ruchkin, D. S., Grafman, J., Cameron, K., \& Berndt, R. S. (2003). Working memory retention systems: A state of activated long-term memory. Behavioral \& Brain Sciences, 26, 709-777.

RUGG, M. D. (1995). ERP studies of memory. In M. D. Rugg \& M. G. H. Coles (Eds.), Electrophysiology of mind: Event-related brain potentials and cognition (Vol. 25, pp. 132-170). Oxford: Oxford University Press.

SCHACTER, D. L., \& CoOPer, L. A. (1993). Implicit and explicit memory for novel visual objects: Structure and function. Journal of Experimental Psychology: Learning, Memory, \& Cognition, 19, 995-1009.

Schacter, D. L., Cooper, L. A., \& Delaney, S. M. (1990). Implicit memory for unfamiliar objects depends on access to structural descriptions. Journal of Experimental Psychology: General, 119, 5-24.

Schon, K., Hasselmo, M. E., LoPresti, M. L., Tricarico, M. D., \& Stern, C. E. (2004). Persistence of parahippocampal representation in the absence of stimulus input enhances long-term encoding: A functional magnetic resonance imaging study of subsequent memory after a delayed match-to-sample task. Journal of Neuroscience, 24, 11088-11097.

Smith, E. E., \& Jonides, J. (1997). Working memory: A view from neuroimaging. Cognitive Psychology, 33, 5-42.

Smith, E. E., Jonides, J., \& KoepPe, R. A. (1996). Dissociating verbal and spatial working memory using PET. Cerebral Cortex, 6, 11-20.

Speckmann, E.-J., CASPERS, H., \& Elger, C. (1984). Neuronal mechanisms underlying the generation of field potentials. In T. Elbert, B. Rockstroh, W. Lutzenberger, \& N. Birbaumer (Eds.), Self-regulation of the brain and behavior (pp. 9-25). Heidelberg: Springer.

Speckmann, E.-J., \& Elger, C. E. (1999). Introduction to the neurophysiological basis of the EEG and DC potentials. In E. Niedermeyer (Eds.), Electroencephalography: Basic principles, clinical applications, and related fields (pp. 15-26). Philadelphia: Lippincott, Williams, \& Wilkins.

Vogel, E. K., \& LUCK, S. J. (2002). Delayed working memory consolidation during the attentional blink. Psychonomic Bulletin \& Review, 9, 739-743.

Wagner,A. D., Schacter, D. L., Rotte, M., Koutstaal, W., Maril,A., DALE, A. M., ET AL. (1998). Building memories: Remembering and forgetting of verbal experiences as predicted by brain activity. Science, 281, 1188-1191.

Williams, P., \& TARR, M. J. (1997). Structural processing and implicit memory for possible and impossible figures. Journal of Experimental Psychology: Learning, Memory, \& Cognition, 23, 1344-1361.

Williams, P., \& TarR, M. J. (1999). Orientation-specific possibility 
priming for novel three-dimensional objects. Perception \& Psychophysics, 61, 963-976.

WOODWARD, A. E., BJorK, R. A., \& JongeWARD, R. H. (1973). Recall and recognition as a function of primary rehearsal. Journal of Verbal Learning \& Verbal Behavior, 12, 608-617.

Zarahn, E., Aguirre, G., \& D'Esposito, M. (1997). A trial-based experimental design for fMRI. NeuroImage, 6, 122-138.

\section{NOTE}

1. Some researchers might consider the transformation of sensory information into representational codes as a WM encoding process rather than a stage of maintenance. This is the case particularly in studies with simple overlearned verbal or spatial stimuli, in which initial WM processing can be rapid and transient (Jolicœur \& Dell'Acqua, 1998; Vogel \& Luck, 2002). However, in studies in which more complex stimuli are used (e.g., the present study; Ranganath, Cohen, \& Brozinsky, 2005), this processing is apparent a few seconds after the stimulus is no longer present. Accordingly, we believe that this processing is more aptly described as a stage of WM maintenance rather than as encoding. Our terminology has the added advantage of clearly distinguishing WM-related processing from sensory processing of a stimulus, which is more directly related to the term encoding.

(Manuscript received December 22, 2005; revision accepted for publication February 20, 2007.) 